\section{A. Marra \\ E. Peuvrel-Disdier \\ A. Wittemann \\ X. Guo \\ M. Ballauff}

\title{
Rheology of dilute and semidilute suspensions of spherical polyelectrolyte brushes
}

\begin{abstract}
The viscosity, $\eta$, of dilute aqueous suspensions of spherical polyelectrolyte brushes is discussed. The spherical polyelectrolyte brushes consist of a solid polystyrene core of 100-nm diameter onto which linear poly(acrylic acid) chains are grafted. The relative viscosity, $\eta / \eta_{\mathrm{S}}$ ( $\eta_{\mathrm{S}}$ is the viscosity of the solvent) of these suspensions is studied as a function of shear rate in the presence of different amounts of added salt. A marked dependence on shear rate is found, in particular when going to higher concentrations. Extrapolation to zero shear rate leads to the
\end{abstract}

relative zero-shear viscosity, $\eta_{0} / \eta_{\mathrm{S}}$, which can be described in terms of an effective volume fraction, $\phi_{\text {eff }}$, for all salt concentrations under consideration. Moreover, the hydrodynamic radii derived from $\phi_{\text {eff }}$ coincide with data obtained by dynamic light scattering in the infinitely dilute regime. Data taken at higher concentrations point to a shrinking of the brush layer owing to mutual interaction.

Keywords Polyelectrolyte B Brush · Colloid · Hydrodynamic radius

\section{Introduction}

Dispersions of polymer particles in water have acquired great technical importance in recent years because of the great number of possible applications [1]. In many cases the stabilization of the suspensions used in technical applications is achieved through affixing linear polymer chains on the surface of the colloidal particles. This steric stabilization [2] can be adjusted within a wide range by changing the grafting density and the interaction of the chains with the surrounding fluid. If charged chains are affixed densely to colloidal polymer particles, spherical polyelectrolyte brushes result [3]. The term brush denotes a layer of linear polymer chains grafted on a surface so densely that the average dimensions of the free chains exceed by far the average distance between the chains on the surface.

Recently, we have demonstrated that spherical polyelectrolyte brushes can be generated by photo-emulsion polymerization $[3,4]$. The structure of these particles is displayed schematically in Fig. 1. The core consists of solid polystyrene onto which linear poly(acrylic acid) (PAA) is grafted. The chains were generated by a grafting-from technique and are thus chemically affixed to the core. The length of the PAA chains can be determined separately and all pertinent parameters of the system, namely the core radius, $R_{\mathrm{c}}$, the contour length, $L_{\mathrm{c}}$, of the grafted chains, and their grafting density, $\sigma$ (number of chains per nanometer squared) can be accurately measured. At sufficiently high pH (above 9), the PAA chains are fully charged $[4,5]$; hence, these particles present nearly ideal model systems for a study of the physics of spherical polyelectrolyte brushes in suspension.

Up to now these systems have been characterized in dilute suspension by measuring their hydrodynamic radius, $R_{\mathrm{H}}[4,5]$. Dynamic light scattering (DLS) is the most convenient tool for the determination of $R_{\mathrm{H}}$ and 


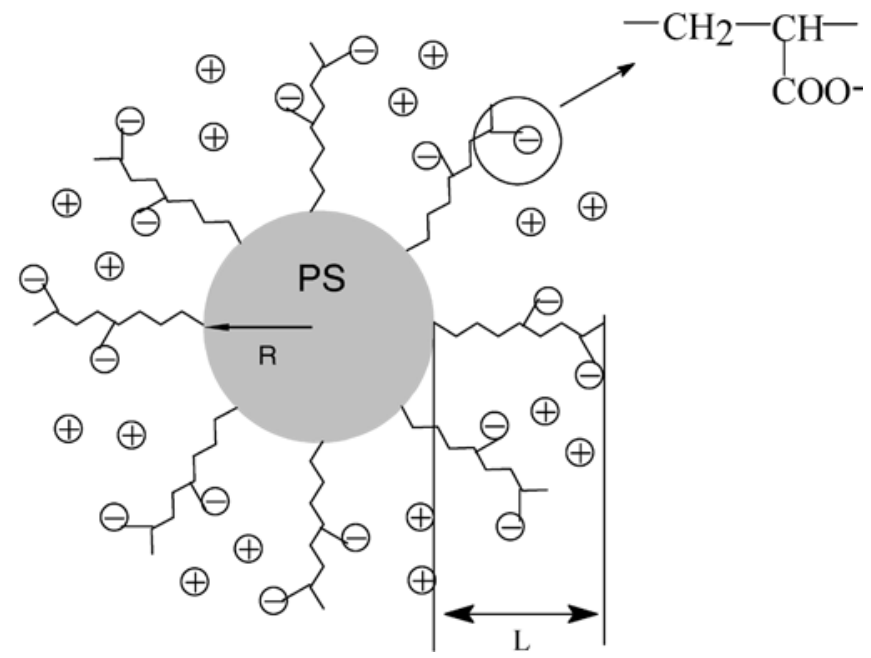

Fig. 1 Scheme of the spherical polyelectrolyte brushes used in this study. Linear chains of poly(acrylic acid) (PPA) are grafted to a core particle of polystyrene. The parameters characterizing the particles are core radius, $R_{\mathrm{c}}, 51.3 \mathrm{~nm}$, contour length of the PAA chains affixed to the surface of the core particles, $L_{\mathrm{c}}, 36 \mathrm{~nm}$, grafting density, $\sigma, 0.129 \mathrm{~nm}^{-2}$

allows this central quantity to be obtained with great accuracy. The $R_{\mathrm{H}}$ thus obtained gives a measure of the overall hydrodynamic dimensions of the noninteracting unperturbed particles as a function of salt concentration and $\mathrm{pH}$. Comprehensive investigations by DLS showed that at low $\mathrm{pH}$ the PAA chains are virtually uncharged and form a dense layer of coiled chains $[4,5]$. At high $\mathrm{pH}$, on the other hand, the fully charged chains are stretched to nearly full length if the salt concentration in the solution is low.

In this communication we present a study of the hydrodynamic radius in the semidilute regime where particles start to interact. The question arises whether $R_{\mathrm{H}}$ obtained by DLS in high dilution may be used to calculate the relative viscosity, $\eta_{0} / \eta_{\mathrm{S}}$, in the dilute regime according to $[6,7]$

$\frac{\eta_{0}}{\eta_{\mathrm{S}}}=1+2.5 \phi_{\mathrm{eff}}+5.9 \phi_{\mathrm{eff}}^{2}$,

where $\eta_{0}$ is the zero-shear viscosity of the suspension, $\eta_{\mathrm{S}}$ is the viscosity of the solvent, and $\phi_{\mathrm{eff}}$ is the volume fraction of particles. Equation (1) is strictly valid for suspensions of hard spheres. Its application to other types of suspensions permits the determination of an effective diameter of interaction and an effective volume fraction, $\phi_{\text {eff. }} \phi_{\text {eff }}$ derives from

$\phi_{\text {eff }}=\frac{N}{V} \frac{4 \pi}{3} R_{\mathrm{H}}^{3}$,

where $N / V$ denotes the number of particles per volume. Hence, $\phi_{\text {eff }}$ may be used to calculate the thickness, $\Delta$, of the surface layer of the particles according to
$\phi_{\mathrm{eff}}=\phi_{\mathrm{c}}\left(1+\frac{\Delta}{a}\right)^{3}=\phi_{\mathrm{c}} k$

where $\phi_{\mathrm{c}}$ denotes the volume fraction of the uncovered particles and $a$ is the core radius.

The flow behavior of particles bearing uncharged chains on their surface seems to be well understood in terms of Eqs. (1) and (2). For example, a recent investigation of particles bearing a thermosensitive network on their surface showed that $R_{\mathrm{H}}$ taken from experimental data by use of Eq (1) coincides with data taken from DLS [8]. Only at the highest frequencies there is a notable difference. Moreover, a recent study of uncharged micelles and star polymers demonstrates that $\phi_{\text {eff }}$ is the decisive parameter for the flow behavior of these systems at zero shear [9].

In the case of charged chains, the comparison of the layer thickness $\Delta$ determined by DLS and rheology should be less straightforward. The presence of charges on the surface of the particles leads to two electroviscous effects under flow, distortion of electric double layer by flow (primary effect) and overlap of double layers of different particles (secondary effect). These effects are well understood by now [2]. Moreover, the presence of salt in the polyelectrolyte system is followed by a profound change of the surface layer (from fully stretched to collapsed chains) as already discussed. This variation of $\Delta$ will drastically change the measured viscosity and has been termed tertiary electroviscous effect by Vaynberg and Wagner [10]. Another issue relevant to the flow behavior of spherical polyelectrolyte brushes is the possible deformation of the brush layer in strong tangential flows. This problem has been examined in detail for planar brushes by Harden et al. [11]. These authors found that there might even be an unexpected dependence of the brush thickness on shear rate. Weakly charged brushes are predicted to expand in strong shear flows, while strongly charged brushes should compress under these conditions.

The first systematic study of the flow behavior of particles with attached polyelectrolyte chains has recently been presented by Fritz et al. [12]. These authors studied suspensions of latex spheres with attached polyelectrolyte chains from the dilute range up to high concentrations. The effective volume fraction was derived by superimposing $\eta_{0} / \eta_{\mathrm{s}}$ taken in the concentrated regime. All $\eta_{0} / \eta_{\mathrm{s}}$ data superimpose on a master curve if the defined effective volume fraction is determined in this way. It has been found, however, that the hydrodynamic radius derived from this effective volume fraction is generally lower than the value derived from DLS. The authors assign this discrepancy to a compression of the brush layer owing to mutual interactions.

The main goal of the present work is the comparison between the hydrodynamic layer thickness as determined through application of Eqs. (1), (2), and (3) with 
the hydrodynamic radius as obtained by DLS. The concentration of added salt was varied over a wide range $(0.1-500 \mathrm{mM})$ to change the thickness of the surface layer from a fully extended state to a collapsed state.

\section{Experimental}

The spherical polyelectrolyte brush termed KPS13 was synthesized according to the method in Refs. [3, 4]. The parameters are $R_{\mathrm{c}}$ $51.3 \mathrm{~nm}, L_{\mathrm{c}} 36 \mathrm{~nm}, \sigma 0.129 \mathrm{~nm}^{-2}$. Suspensions were prepared by serum replacement. Colloidal particles were retained in a stirred cell (Amicon) by way of a nitrocellulose membrane (Schleicher \& Schuell) with $100-\mathrm{nm}$ pores. The original solvent (bidistilled water) was completely replaced by a washing solution having defined $\mathrm{pH}$ and ionic strength. The $\mathrm{pH}$ of the washing solution was adjusted to 10 using $\mathrm{NaOH}$ and the ionic strength was adjusted by adding $\mathrm{NaCl}$ or $\mathrm{NaOH}$. Six different ionic strengths were investigated $(0.1$, $1,10,50,100$, and $500 \mathrm{mM}$ ). It should be noted that these particles are stable up to $3 \mathrm{M}$ monovalent salt [5]. The content of solid of the respective suspensions was determined gravimetrically. The density of the suspensions was measured using a DMA 60 apparatus supplied by Paar (Graz, Austria).

DLS was done using a Peters ALV 4000 light scattering goniometer.

Two different types of viscometers were used in this study: Ubbelohde viscometers no. $1 \quad\left(k=0.00370 \mathrm{~s}^{-1}\right)$ and no. 2 $\left(k=0.00498 \mathrm{~s}^{-1}\right)$; a Bohlin CV0120 viscometer equipped with a double-gap DG 40-50 Couette system made from stainless steel. All the viscometers were calibrated by use of Cannon Instruments standard solutions. The results obtained using the Ubbelohde viscometers were corrected for kinetic effects by the HagenbachCouette equation [13]. The Couette system served for measurements at shear rates of 1,10 , and $100 \mathrm{~s}^{-1}$ whereas the Ubbelohde viscometers were used to characterize the suspensions at $900 \mathrm{~s}^{-1}$ (no. 2) and $1,200 \mathrm{~s}^{-1}$ (no. 1). The average shear rate, $\langle\dot{\gamma}\rangle$, in the Ubbelohde viscometers was calculated according to the formula

$\langle\dot{\gamma}\rangle=3 \frac{V}{\pi R^{3}} \frac{1}{t}$,

where $V$ is the volume of fluid passed through the capillary with radius $R$ in time $t$.

All measurements (DLS and viscosity) were carried out at $20 \pm 0.05^{\circ} \mathrm{C}$.

\section{Results and discussion}

The variation of the hydrodynamic radius as determined by DLS versus the concentration of added salt is given in Table 1. The marked dependence is typical for polyelectrolyte brushes and has recently been traced back to the confinement of the counterions within the brush [5]. At low concentrations of added salt, the osmotic pressure of the counterions leads to a strong stretching of the polyelectrolyte chains. In this limit (osmotic brush) the chains are stretched to nearly full length. On the other hand, a high concentration of added salt leads to a strong screening of electrostatic interactions within the brush. Hence, the chains adopt the usual random-coil configuration of an uncharged polymer. All data could be described by a simple model based on the classical two-parameter theory of linear polymers together with
Table 1 Hydrodynamic radius as a function of the ionic strength indicated in the first column. The second column contains data obtained by dynamic light scattering $(D L S)$ at high dilution, whereas the last column contains data obtained from viscosity measurements according to Eqs. (1), (2), and (3)

\begin{tabular}{ccc}
\hline $\begin{array}{l}\text { Ionic strength } \\
(\mathrm{mM} \text { of } \mathrm{NaCl})\end{array}$ & $\begin{array}{l}\text { DLS radius } \\
(\mathrm{nm})\end{array}$ & $\begin{array}{l}\text { Hydrodynamic } \\
\text { radius }(\mathrm{nm})\end{array}$ \\
\hline 0.1 & 191 & 186 \\
1 & 163 & 158 \\
10 & 120 & 120 \\
50 & 117 & 110 \\
100 & 106 & 105 \\
500 & 102 & 103 \\
\hline
\end{tabular}

the Daoud-Cotton model modified by Hariharan et al. [14]. In the following the hydrodynamic radii measured at different ionic strengths (by DLS) is used as an input parameter and is compared to the hydrodynamic radii derived from $\Delta$ (determined from viscosity measurements cf. Eqs. 1, 2, 3). Evidently, the low particle concentrations typical for DLS exclude all effects of mutual interactions. Hence, $R_{\mathrm{H}}$ taken from DLS refers to the limit of infinite dilution. No disturbances by mutual interactions of the particles need to be taken into account.

The combination of the viscometers used in the present study allows the shear-rate dependence of the suspensions of the spherical polyelectrolyte brushes to be tested over 3 decades. A typical result of the resulting specific viscosity $\left(\eta_{\mathrm{sp}}=\eta / \eta_{\mathrm{S}}-1\right)$ is displayed in Fig. 2 as a function of the shear rate, $\dot{\gamma}$. The parameter varying for the different curves is the concentration of particles. This figure clearly shows a Newtonian behavior of the suspensions in the highly dilute regime and a shearthinning behavior as the concentration increases.

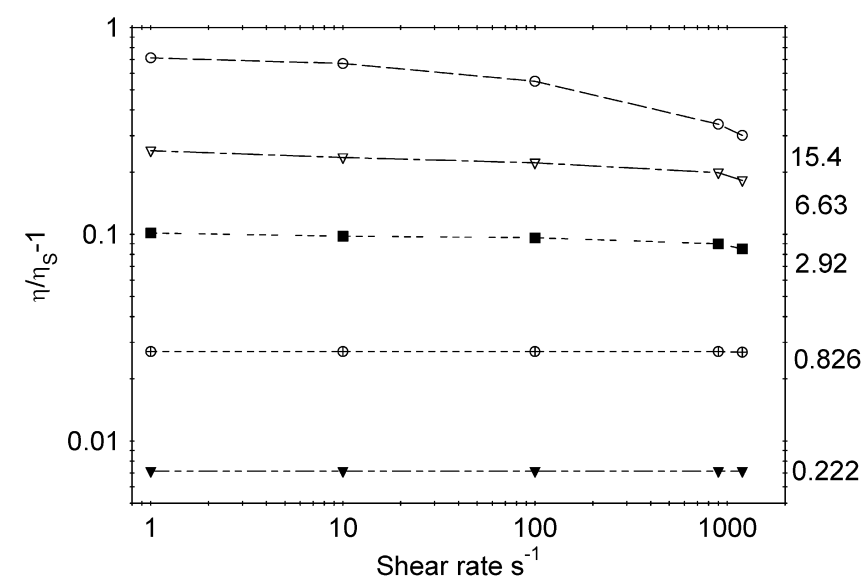

Fig. 2 Dependence of the specific viscosity on the shear rate for different volume fractions, $\phi_{\mathrm{c}}\left(\times 10^{3}\right)$, indicated in the graph. All measurements were done at a ionic strength of $10 \mathrm{mM}$ and a $\mathrm{pH}$ of 10 
The strong effect of shear rate is also shown in Fig. 3, where the relative viscosity is plotted as a function of the effective volume fraction (same data as Fig. 2). $\phi_{\text {eff }}$ was calculated from the $R_{\mathrm{H}}$ taken from DLS (Table 1). The marked differences of $\eta / \eta_{\mathrm{S}}$ measured at different shear rates are directly evident when increasing the effective volume fraction of the particles.

The small dependence of $\eta / \eta_{\mathrm{S}}$ on the shear rate in the highly dilute regime points to the fact that the particles are not strongly deformed in this regime. Shear thinning becomes apparent only if the particles start to interact. It is interesting to note that at the low effective volume fractions investigated, the average distance between particles is still much larger than their diameter. Hence, the coronae of the different particles do not overlap, but mutual interaction is mainly due to electrostatic repulsion.

The relative zero-shear viscosity can be obtained from these data by extrapolation of the viscosity curves to vanishing shear rate. Here we used the procedure suggested by Golub [15] for linear polymer chains. This theory predicts that the shear rate dependence of the viscosity can be expressed as follows:

$\frac{\eta_{\mathrm{sp}}}{c}=\frac{\eta_{\mathrm{sp}}^{0}}{c}\left[1-k\left(\frac{\eta_{\mathrm{sp}}}{c} \dot{\gamma}\right)^{1 / 2}\right]$,

where $\eta_{\mathrm{sp}}^{0}$ is the specific viscosity at zero shear rate, $k$ an empirical constant, and $c$ the concentration of particles in suspension (grams per milliliter). Such a plot for the data discussed already is shown in Fig. 4. The parameter varying for the different curves is the effective volume

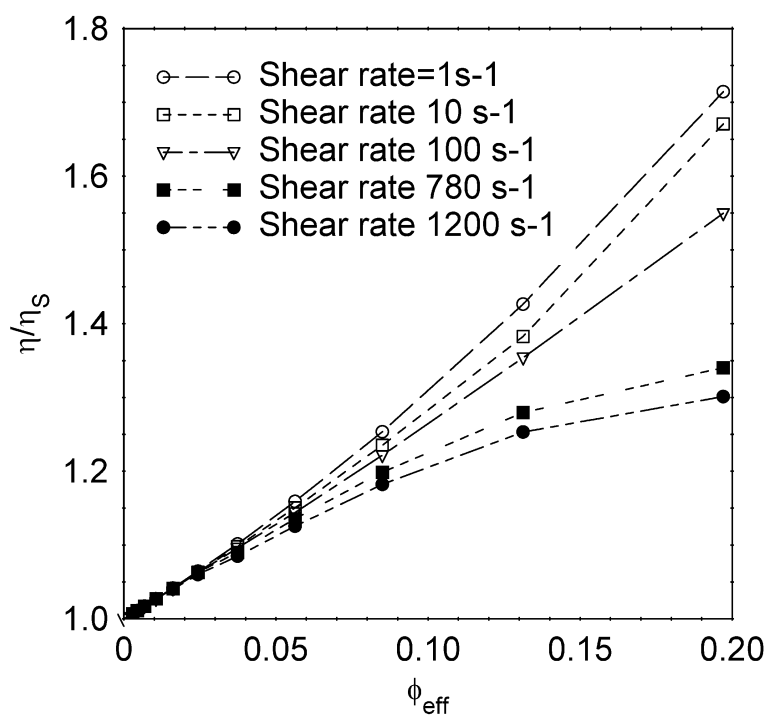

Fig. 3 Relative viscosity, $\eta / \eta_{\mathrm{S}}$, as a function of the effective volume fraction, $\phi_{\text {eff. }}$ The concentration of added salt is $10 \mathrm{mM}$ and the $\mathrm{pH}$ is 10. The effective volume fraction was calculated via Eq. (2) using the hydrodynamic radii, $R_{\mathrm{H}}$, obtained from dynamic light scattering. The shear rate for each set of data is indicated on the graph fraction of the particles. Good linearity is seen for all concentrations, which indicates the validity of the procedure for spherical brushes. Similar plots were obtained for all other salinities investigated in this study.

The extrapolation to vanishing shear rate permits the determination of the relative zero-shear viscosity. The variation of the relative zero-shear viscosity is shown in Fig. 5 as a function of $\phi_{\text {eff }}$ for different concentrations of added salt. Fig. 5a displays all data, whereas only an enlarged view of the dilute regime is given in Fig. 5b. Evidently, all data obtained for the different concentrations of added salt form a master curve.

The dilute regime $\left(\phi_{\mathrm{eff}}<0.06\right)$ can now be used to compare the hydrodynamic radii determined from viscosity data at higher concentrations than those derived from DLS in the infinitely dilute regime. If the different hydrodynamic radii agree, Eq. (1) should provide a perfect fit of the zero-shear viscosities in the dilute regime. This is exactly what is seen in Fig. 5b, where the prediction of Eq. (1) lies directly on the master curve. This shows that the model of hard spheres can successfully be mapped on the present data taken in the dilute regime. Hence, we can conclude that no change of the hydrodynamic volume has taken place when increasing the effective volume fraction up to moderate values in which binary interactions of the particles become nonnegligible.

Note that this conclusion holds true over the entire range of concentration of added salt. The present set of experimental data ranges from a nearly collapsed state of the attached chains (high ionic strength) to a nearly

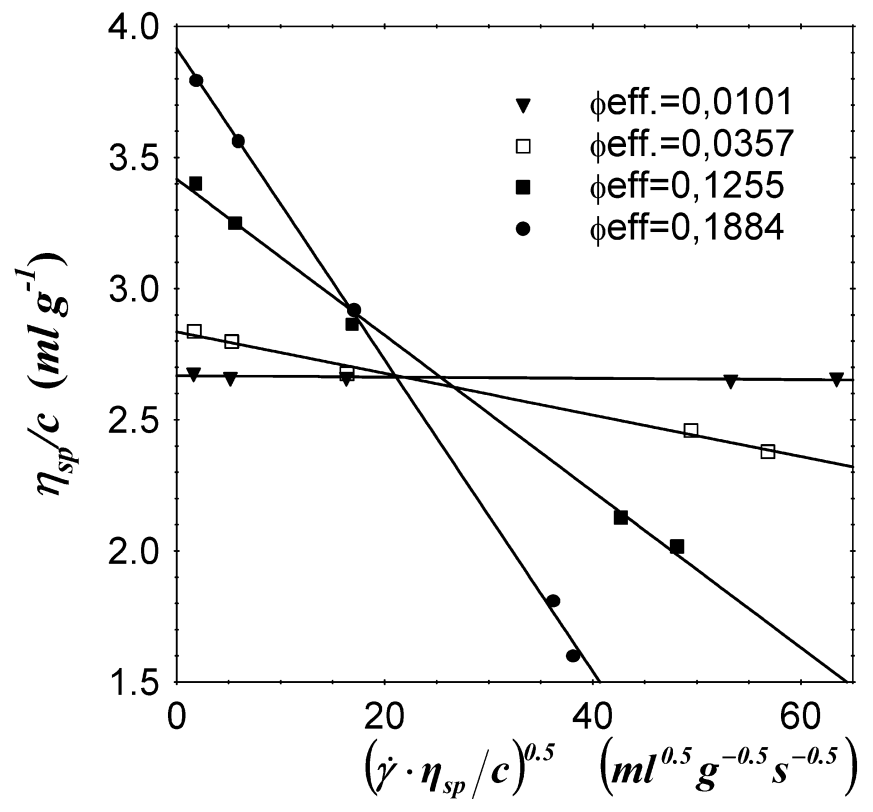

Fig. 4 Extrapolation of the reduced viscosity, $\eta_{\mathrm{sp}} / c$, to vanishing shear rates according to Eq. (4). The reduced viscosity is plotted versus $\left(\dot{\gamma} \eta_{\mathrm{sp}} / c\right)^{0.5}$ 

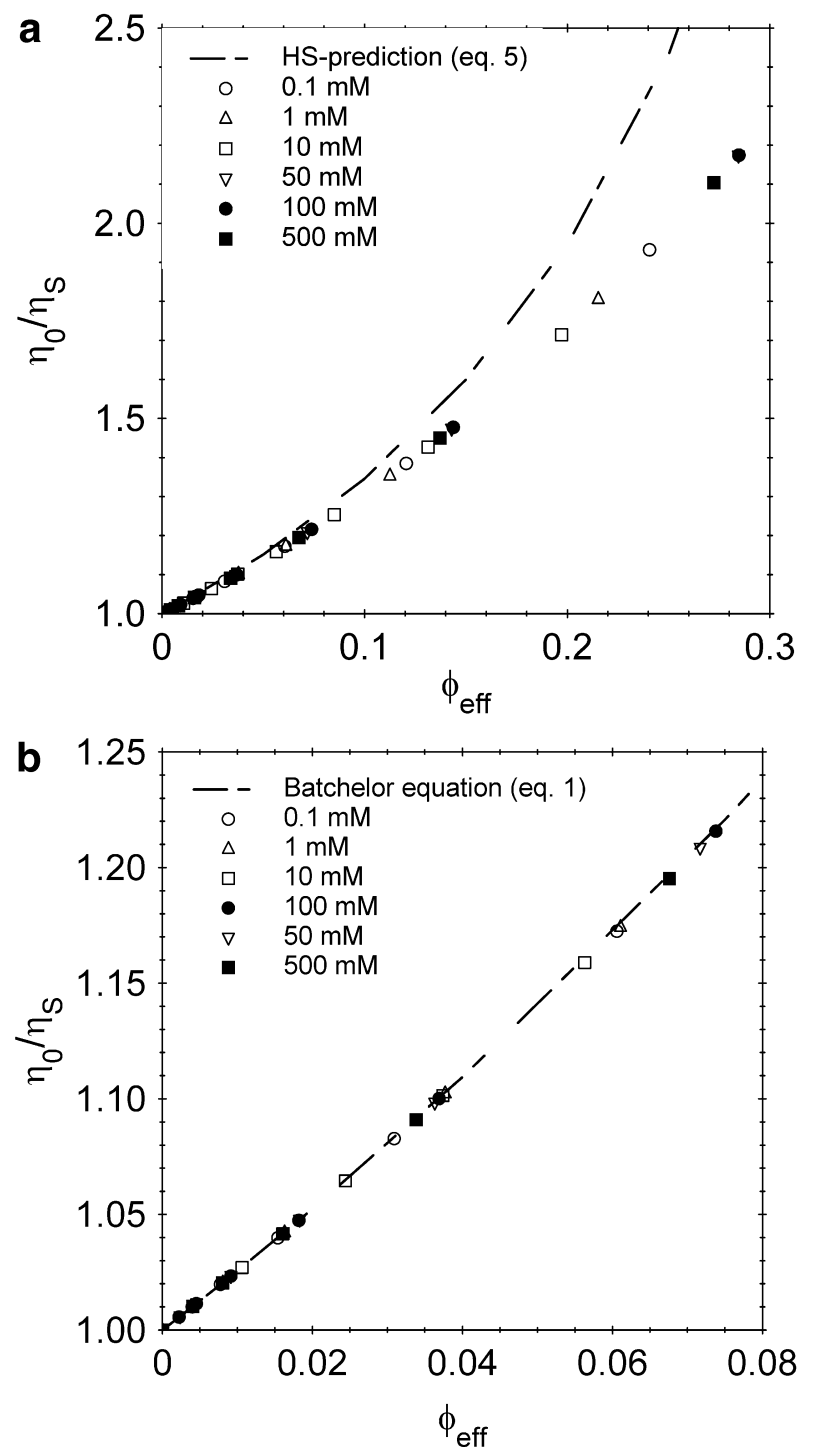

Fig. 5a,b Summary of all data. Relative viscosity, $\eta_{0} / \eta_{\mathrm{S}}$, extrapolated to vanishing shear rates as a function of the effective volume fraction calculated using Eq. (2) and the hydrodynamic radii obtained from dynamic light scattering. a The entire set of data; b an enlarged view of the dilute regime

fully extended state (low salt concentration). This underscores that the hydrodynamic radius is a valid measure for the extension of the chains grafted to the surface. Moreover, the full agreement demonstrates that the particles interact solely by mutual repulsion, there is no sign for residual attraction that would immediately show up in an enormous increase of the coefficient of $\phi_{\text {eff }}$ (Batchelor coefficient) in Eq. (1). Such a marked upturn of $\eta_{0} / \eta_{\mathrm{S}}$ was observed in a previous study on thermosensitive particles [8].
The dashed line in Fig. 5a displays the master curve derived for hard spheres by Meeker et al. [16]:

$\frac{\eta_{0}}{\eta_{\mathrm{S}}}=\left(1-\frac{\phi}{\phi_{\mathrm{m}}}\right)^{-[\eta] \phi_{\mathrm{m}}}$,

with $[\eta]=3.2$ and $\phi_{\mathrm{m}}=0.55$.

This equation provides a good description of the relative viscosity of the spherical polyelectrolyte brushes in the dilute regime. It is clear, however, that this equation strongly overestimates the viscosity when going to higher effective volume fractions. This may be traced back to a shrinkage of the polyelectrolyte brushes when increasing the concentration when many particles start to interact. It should be noted that this effect is not due to an increase of the ionic strength brought about by an increasing number of particles since all concentrations were prepared separately and that the ionic strength was controlled for each suspension.

The slight shrinkage of the particles observed here has also been reported in the recent investigation of Fritz et al. [12]. These workers found that the effective volume fraction derived from viscosity data taken at high concentrations is smaller than the values derived from DLS. Evidently, the present results show that strongly interacting spherical polyelectrolyte brushes start to shrink beyond effective volume fractions of 0.1 .

\section{Conclusion}

A rheological study of the dilute and semidilute regime of the spherical polyelectrolyte brushes shown in Fig. 1 has been presented. The data demonstrate that mutual interactions lead to a marked dependence of the measured viscosity as a function of shear rate. Data of the relative viscosity obtained by extrapolation to vanishing shear rates can be described in terms of the hydrodynamic radius and the effective volume fraction derived from DLS. The hydrodynamic radii obtained by DLS at high dilution therefore provides a valid description of $\eta_{0} / \eta_{\mathrm{S}}$ at concentrations where binary interactions of the spheres are important. These data confirm that the hydrodynamic radius determined from the relative zero-shear viscosity is a valid measure for the extension of the shell thickness dimension under quiescent conditions in agreement with recent studies $[4,5]$. For $\phi_{\text {eff }}>0.1$, the data show an onset of a shrinkage of the polyelectrolyte brushes.

Acknowledgements Financial support by the European Community (HUSC) and by the Deutsche Forschungsgemeinschaft are gratefully acknowledged. N. Willenbacher is thanked for making available Ref. [12] prior to appearance. The authors are indebted to S. Guido, N. Grizzuti, and G. Marrucci, Chemical Engineering department of Naples, for the use of the Bohlin rheometer and for their technical support during the experiments. 


\section{References}

1. Distler D (1999). Waessrige Polymerdispersionen. Wiley-VCH, New York

2. Russel WB, Saville DA, Schowalter WR (1989). Colloidal dispersions. Cambridge University Press, Cambridge

3. Guo X, Weiss A, Ballauff M (1999) Macromolecules 32:6043

4. Guo X, Ballauff M (2000) Langmuir $16: 8719$

5. Guo X, Ballauff M (2001) Phys Rev E 64:051406
6. Batchelor GK (1977) J Fluid Mech 83:97

7. Brady JF, Vicic M (1995) J Rheol 39:545

8. Deike I, Ballauff M, Willenbacher N, Weiss A (2001) J Rheol 45:709

9. Vlassopoulos D, Fytas G, Pispas S, Hadjichristidis N (2001) Physica B 296:184

10. Vaynberg A, Wagner NJ (2001) J Rheol 45:451

11. Harden JL, Borisov OV, Cates ME (1997) Macromolecules 30:1179
12. Fritz G, Schädler V, Willenbacher N, Wagner NJ (2002) Langmuir 18:6381

13. Cannon MR, Manning RE, Bell JD (1969) Anal Chem 32:355

14. Hariharan R, Biver C, Russel WB (1998) Macromolecules 31:7514

15. Golub MA (1957) Can J Chem 35:381

16. Meeker SP, Poon WCK, Pusey PN (1997) Phys Rev E 55:5718 